\section{Lighting Up Cells with Quantum Dots}

\author{
Andy Watson, Xingyong Wu, and \\ Marcel Bruchez \\ Quantum Dot Corporation, Hayward, CA, USA
}

worldwide energy crisis. Research in photoelectrochemistry (e.g., solar energy conversion) was tapping the semiconductor/liquid interface to exploit the advantageous surface areato-volume ratio of nanocrystal particles for energy generation. Seminal developments arose in the early 1980s from two laboratories, worlds apart: Dr. Louis Brus at Bell Laboratories, and Drs. Alexander Efros and A.I. Ekimov of the Yoffe Institute in St. Petersburg (then Leningrad) in the former Soviet Union. Two of the Bell Labs scientists-Dr. Moungi Bawendi and Dr. Paul Alivisatos - moved to MIT and UC Berkeley, respectively, and continued to investigate quantum dot optical properties. They and other researchers found ways to make the quantum dots water-soluble $(5,6)$. This pioneering work paved the way for the current quantum dot conjugates.

\section{QUANTUM DOT BIOCONJUGATES}

beling reagents. These nanometer-sized semiconductor crystals can be attached to biomolecules and can be used in many existing immunostaining applications. Recent work has demonstrated the dramatic benefits of quantum dots, including long-term photostability, high brightness, multi-target labeling with several colors, and single-source excitation for all colors of quantum dots. New avenues of research are being enabled by using quantum dot conjugates in live cell analysis. Here the underlying technology is explained, with a description of the use of quantum dots in microscopy and the implications for fluorescence imaging methods.

\section{A SHORT HISTORY}

Victorian-era glass-melt recipes for red and orange colors contain, essentially, dilute concentrations of nanocrystallites (from zinc and cadmium sulfides and selenides). These nineteenth-century chemists created these nanocrystals spontaneously (and unknowingly) during the glass manufacturing process. These glasses were used to create colored windows. However, these richly hued progenitors lacked the fluorescence of the quantum dots currently used for biological applications. Modern quantum dot technology traces its origins, in part, to the mid-1970s quest for new answers to meet the
Quantum dot bioconjugates are made from a nanometerscale crystal of semiconductor material, cadmium selenide, which has been coated with an additional semiconductor shell ( $\mathrm{ZnS})$ to improve the optical properties of the material. This core-shell material (Figure 1A) is further coated with a polymer shell (Figure 1B) $(9,18,20)$ or other ligands $(1,5,6,12,13$, $16,19)$ that allows the materials to be conjugated to biological molecules and to retain their optical properties. We have extensively tested many of these water solubilization schemes and have found that the polymer shell approach used in the method. This polymer shell can then be directly coupled to streptavidin or other biomolecules. The Qdot ${ }^{\mathrm{TM}} 605$ Streptavidin Conjugate (18) is the size of a large macromolecule or protein $(10-15 \mathrm{~nm})$.

These quantum dot conjugates are used as a colloidal suspension, stable in many common buffers. The conjugates may be used in the same way as other labeled conjugates for in vitro uses such as tissue staining, cell surface staining, and intracellular staining (20). As quantum dot conjugates are multivalent (i.e., more than one functional biomolecule is attached to a single quantum dot), they are typically used at saturating conditions to avoid cross-linking of antigens. work described here is the most versatile and best performing
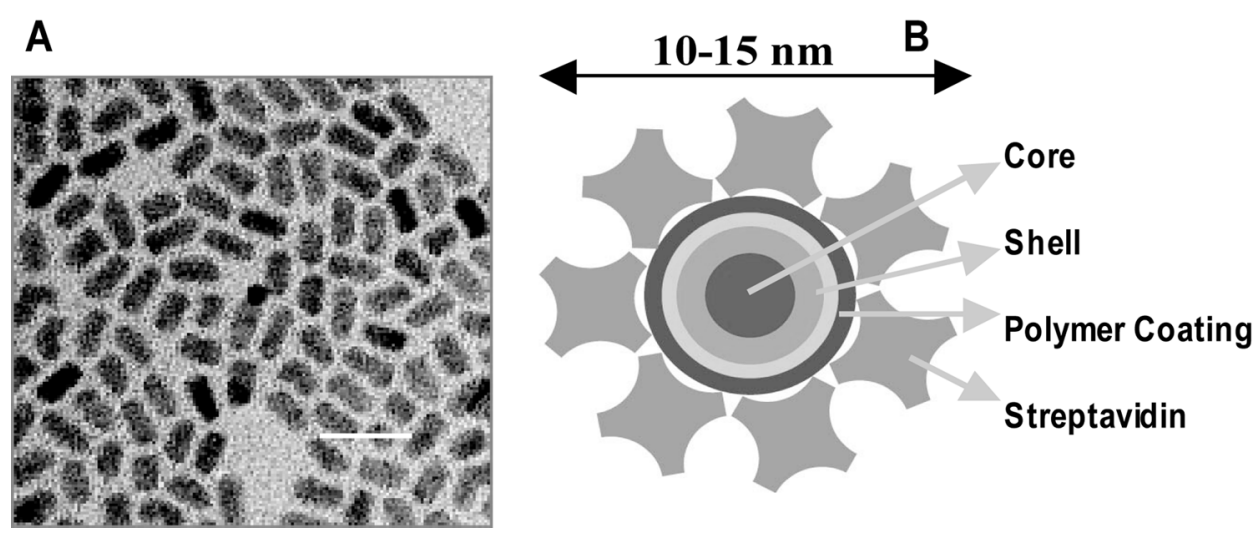

Figure 1. Qdot conjugate structure. (A) Transmission electron microscope image of core-shell Qdot Nanoparticles at $200000 x$ magnification. Scale bar $=20 \mathrm{~nm}$. (B) Schematic of the overall structure of a Qdot Streptavidin Conjugate Probe. The layers represent the distinct structural elements of the Qdot nanocrystal conjugates and are roughly to scale. 


\section{OPTICAL PROPERTIES AND DETECTION}

Quantum dots emit light in a similar manner as a bulk semiconductor light-emitting diode (LED). However, LEDs are electrically activated, whereas quantum dot bioconjugates are activated by the absorption of a photon of light (Figure 2A). This absorbed photon of light creates an electron-hole pair - this electron hole quickly recombines leading to emission of a lower-energy photon. In a given semiconductor material (e.g., CdSe), the energy of the emitted photon is determined by the size of the quantum confinement (or the size of the quantum dot). As the energy of the emitted photon determines the emission wavelength, this means that quantum dots just a few ångstroms different in size emit different wavelengths of light (Figure 2B). This quantum mechanical physics $(2,7,8,10,14,15,17)$ can be simply summarized: smaller quantum dots emit blue light, and larger ones emit red light. Quantum dots may also be synthesized using different core semiconductor materials such as cadmium telluride, indium phosphide, or indium arsenide, and this changes the range of emission wavelengths; however, none of these materials has yet been shown to be a useful bioconjugate.

Stokes shift is the difference between the maximum absorbance and emission wavelengths of a dye. Typical fluorescent dyes have excitation and emission spectra with a relatively small Stokes shift, which means that the optimal excitation wavelength is close to the emission peak. Filter sets used with fluorescence dyes reflect this characteristic. Quantum dots have absorbance spectra that increase dramatically to the blue of the emission (Figure 3). These unique spectral properties are due to the semiconductor that makes up the core of the quantum dot conjugates, which gives rise to both the absorbance and emission properties of the materials. In spite of the broad absorbance, the emission wavelength is independent of the excitation wavelength; so, whether exciting at 595 or $400 \mathrm{~nm}$, the shape of the emission remains the same, while the intensity is approximately 5-fold higher with 400 nm excitation.

Qdot 605 Streptavidin Conjugates have an extinction coefficient of approximately $650000 \mathrm{M}^{-1} \mathrm{~cm}^{-1}$ at $600 \mathrm{~nm}$, increasing to around $3500000 \mathrm{M}^{-1} \mathrm{~cm}^{-1}$ at $400 \mathrm{~nm}$, and even higher at shorter wavelengths (compared to fluorescein, which is around $80000 \mathrm{M}^{-1} \mathrm{~cm}^{-1}$ at its peak). In practice, this means that much more of the excitation light is absorbed than with organic dyes, leading to brighter signals. Alternatively, lower-power excitation can be used to reduce photo-induced effects on the sample being analyzed.

The width of the emission spectrum of a quantum dot solution can be very narrow (less than $27 \mathrm{~nm}$ full width at half maximum in commercial products). The emission spectrum is also symmetrical and does not exhibit the "red tails" associated with organic dye molecules, which have very asymmetric emission peaks that tail into the red. These narrow emission spectra allow many quantum dot colors to be analyzed simultaneously, with minimal overlap between spectrally adjacent colors. The width of the emission spectrum is caused by both the spectral width of single quantum dots and by slight size differences between the quantum dots in a given batch. The ultimate limit of the emission line width is around $15 \mathrm{~nm}$, which 


\section{Biedmaging}

is the spectral emission width of a single quantum dot (10).

The absorbance and excitation at shorter wavelengths, with fixed emission, result in a large "apparent Stokes shift." Quantum dots do not have a fixed value for Stokes shift because absorbance increases continuously with decreased wavelength. A large Stokes shift improves sensitivity by reducing autofluorescence (i.e., fluorescence generated by the biological sample itself; normally greatest at wavelengths close to the excitation wavelength). This large apparent Stokes shift reduces the demands on the optical system, allowing higher performing and less expensive instrumentation.

Quantum yield (i.e., the number of photons emitted divided by number of photons absorbed) is a critical property of any fluorescent conjugate. With a low quantum yield, the efficiency is very low, and so the signal intensity and sensitivity can be poor. The quantum yield of most organic dye molecules is reduced, sometimes to zero, upon conjugation to biomolecules. Commercial quantum dot conjugates have quantum yields up to $80 \%$ and are typically unaffected by attachment to biomolecules.

\section{EPIFLUORESCENCE MICROSCOPY}

Quantum dots can be used immediately in existing epifluorescence microscopes that are equipped with dye-optimized filter sets (Figure 4). The broad absorbance spectrum of the quantum dot allows a unique possibility in lamp-based fluorescent microscopes. The integrated absorbance across a broader excitation band is substantially higher than any single wavelength value. Using a lamp with a short-pass filter allows highly efficient excitation of the quantum dots, as a much larger proportion of the lamp energy is utilized. To achieve the maximum signal from the Qdot 605 Streptavidin Conjugate, a custom excitation filter and dichroic mirror-ex E460SPUV, dichroic 475DCXRU (Chroma Technology, Brattleboro, VT, USA) — give enhanced signal (Figure 4). The narrow emission spectra of quantum dots allow the use of narrower emission filters compared to dye filter sets. We use 20-nm bandpass filters, centered around the emission peaks of the quantum dots. The narrow bandpass filters reject more of the sample autofluorescence (increasing signal-to-noise ratio).

In an epifluorescence microscope, many colors of quantum dots can be detected simultaneously by eye using a fixed short-pass excitation filter and dichroic mirror, with a longpass emission filter - it is quite striking actually to visualize several quantum dot colors simultaneously without switching filter cubes. A simple automated multicolor system for quantum dot detection comprises a fixed short-pass excitation filter and dichroic mirror, and a CCD camera with a filter wheel. The emission filters in the wheel are selected to be centered around the quantum dot emission wavelengths. Figure 6 shows an example composite image using this approach.

The quantum dot emission is very photostable (Figure 5) This makes the quantum dots very easy to use, as samples can be scanned without concerns of losing the fluorescence image. Quantitative analysis may also be simplified, as photobleaching effects are removed. High photostability also allows the use of long integration times, to improve sensitivity and signal-to-noise ratio. In our laboratory, we regularly leave slides on the microscope under continuous illumination, for easy demonstration.

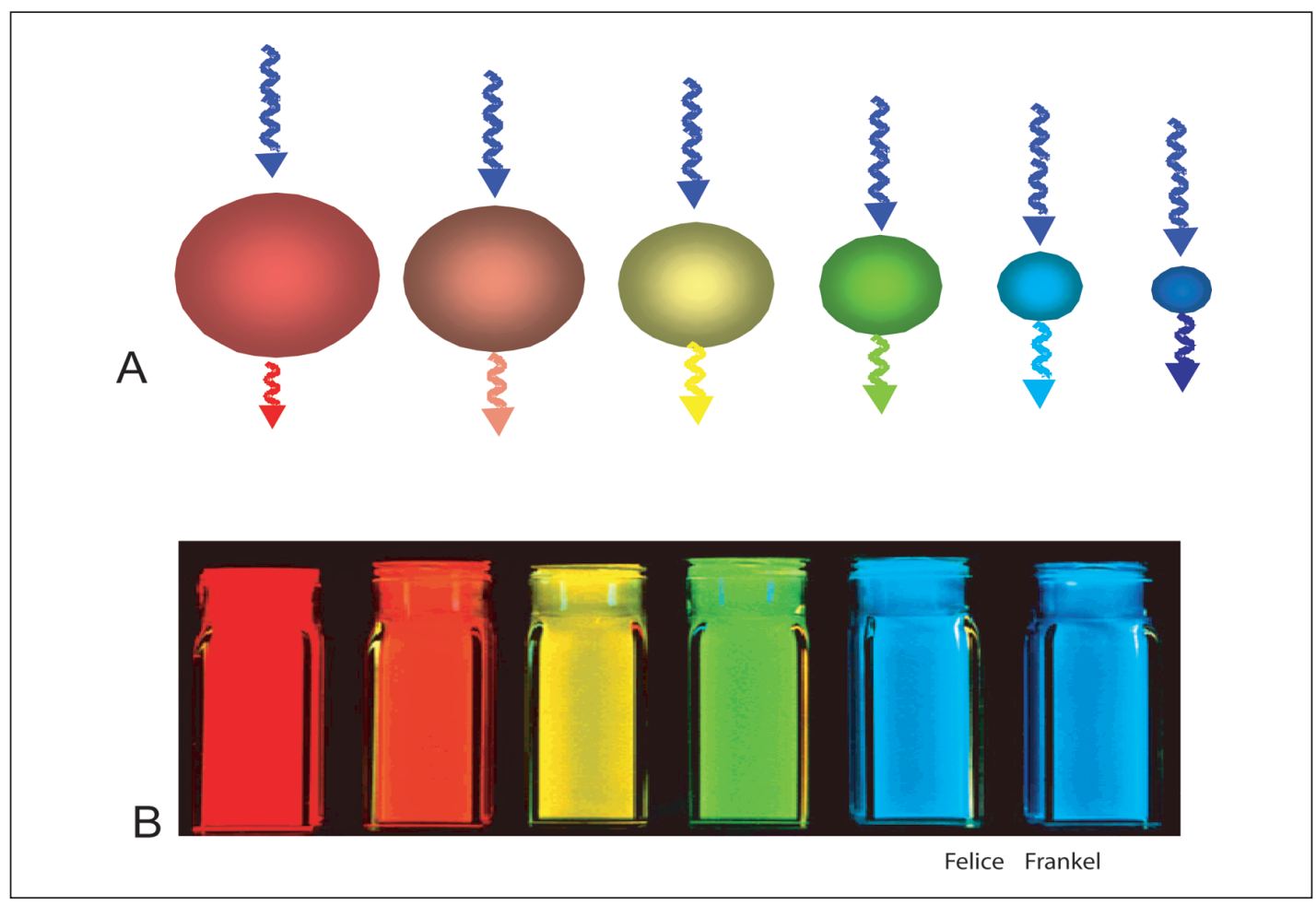

Figure 2. Different colors of quantum dots. (A) Nanocrystals absorb light and then re-emit the light in a different color; the size of the nanocrystal at the ångstrom scale) determines the color. (B) Six different quantum dot solutions are shown excited with the same long-wave UV lamp. 


\section{Biedmaging}

\section{CONFOCAL MICROSCOPY}

Quantum dot bioconjugates are ideal for confocal microscopy. The broad excitation allows the use of any of several commonly available lasers. For example, Qdot 605 conjugates are efficiently excited by $568-\mathrm{nm}, 532-\mathrm{nm}, 488-\mathrm{nm}$, $457-\mathrm{nm}, 405-\mathrm{nm}$, and UV lasers. The $405-\mathrm{nm}$ source is particularly desirable because it is a very inexpensive laser, and the quantum dots have very high absorbance at $405 \mathrm{~nm}$. Several manufacturers have recently introduced lower-cost confocal microscopes that incorporate a $405-\mathrm{nm}$ laser source. Quantum dots allow true multicolor confocal analysis, as all of the quantum dots can be excited by a single laser source. This removes issues such as accurate laser alignment or excitation chromatic effects. The high photostability exhibited by the quantum dots (Figure 5) is especially valuable for confocal microscopy. When data are being gathered from one image plane, other image planes remain illuminated by the laser source. In the case of organic dyes, these out-of-plane images may be unintentionally photobleached, leading to inaccurate measurements. Out-of-plane quantum dots remain highly luminescent.
In confocal microscopes, a very high power density may be achieved through the use of a highly focused laser. Leaving photobleaching effects aside, the light emitted from an organic dye increases with increasing power density only up to the point of saturation of the dye, according to the "excited state lifetime" of the dye (i.e., the time between absorption of a photon and emission of a photon from the dye molecule). In the excited state, the organic dye is unable to absorb another photon. Therefore, saturation occurs at high power densities. The excited state lifetime of quantum dots is approximately $12 \mathrm{~ns}$, compared to around $2 \mathrm{~ns}$ for an organic dye. Unexpectedly, however, we have not seen quantum dot saturation at expected power densities, an effect not yet fully understood. Blinking, where single quantum dots change from a "dark" to an "active" state, has been reported (10) and could be expected to reduce the signal from a quantum dot assay. This effect is strongly dependent on the quality of the synthesis of the quantum dots, in particular the thickness of the inorganic shell, and does not appear to be a problem with current commercial quantum dot conjugates.

Quantum dots have been reported to be efficiently excited in a dual- or multi-photon mode (4). However, we are not yet

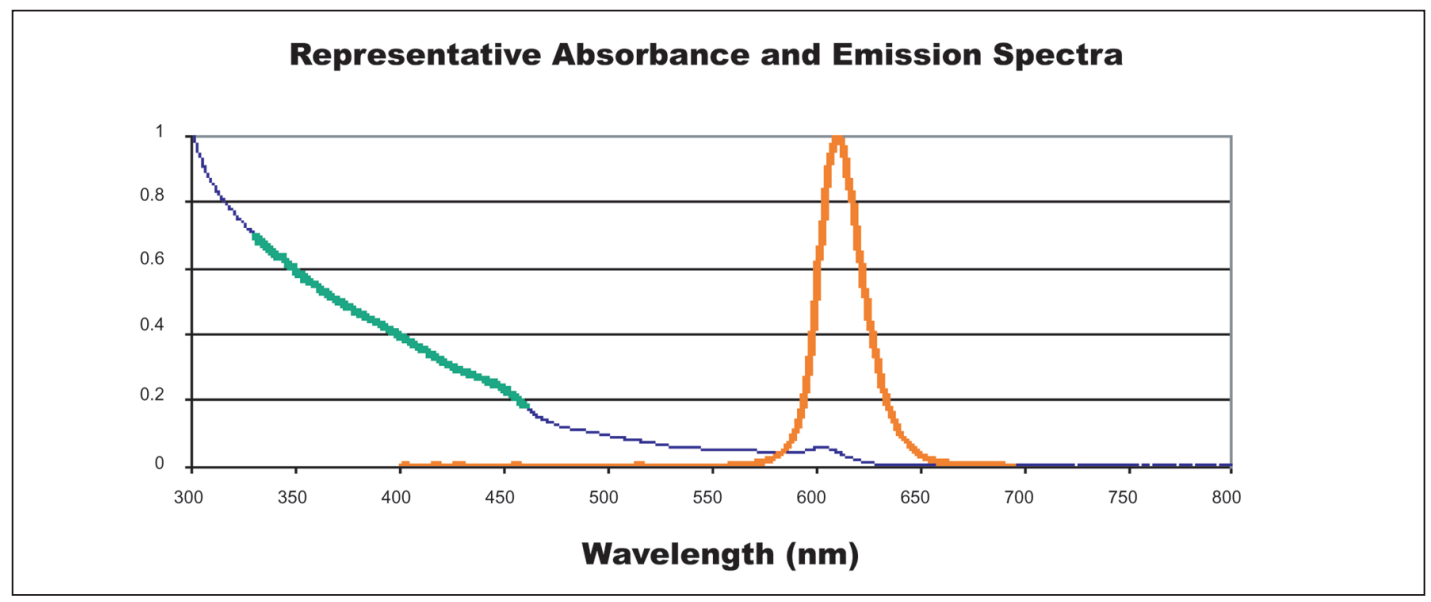

Figure 3. Absorbance (blue and green) and emission (orange) spectra of a typical Qdot 605 streptavidin conjugate. The green line represents a broad window of absorbance that will excite the materials more efficiently than a single-wavelength excitation. Such excitation can be achieved through the use of a shortpass excitation filter.

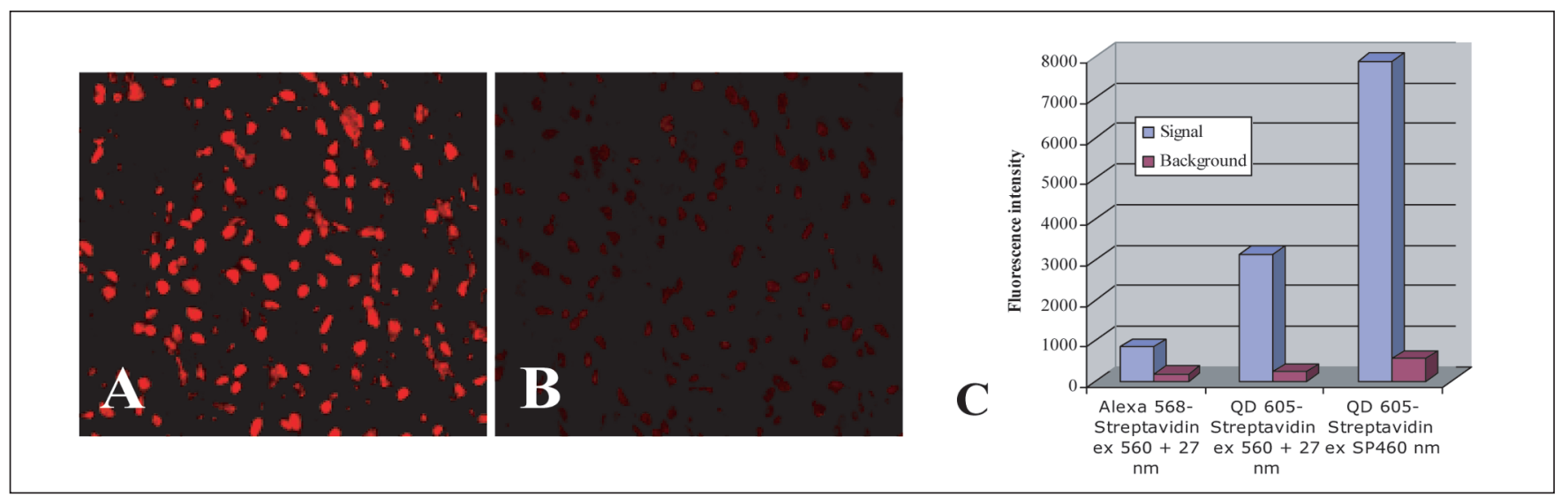

Figure 4. Fluorescent intensity comparison between Qdot 605 streptavidin conjugate and Alexa Fluor ${ }^{\circledR} 568$ streptavidin conjugate. Mouse kidney sections were incubated sequentially with human anti-nuclear antigens antibodies, biotinylated goat anti-human IgG and Qdot 605 streptavidin conjugate (A) or Alexa Fluor 568 (Molecular Probes, Eugene, OR, USA) streptavidin conjugates (B). Both conjugates were detected and imaged with their optimal filter sets. Qdot 605 streptavidin conjugate showed strong staining of the nuclei (A), while Alexa Fluor 568 conjugate exhibited much weaker signals (B) when the same gain and exposure times were used to capture images with a CCD camera. A replacement excitation filter allowed further enhancement of the signal intensity (C). 


\section{Bielmaging}
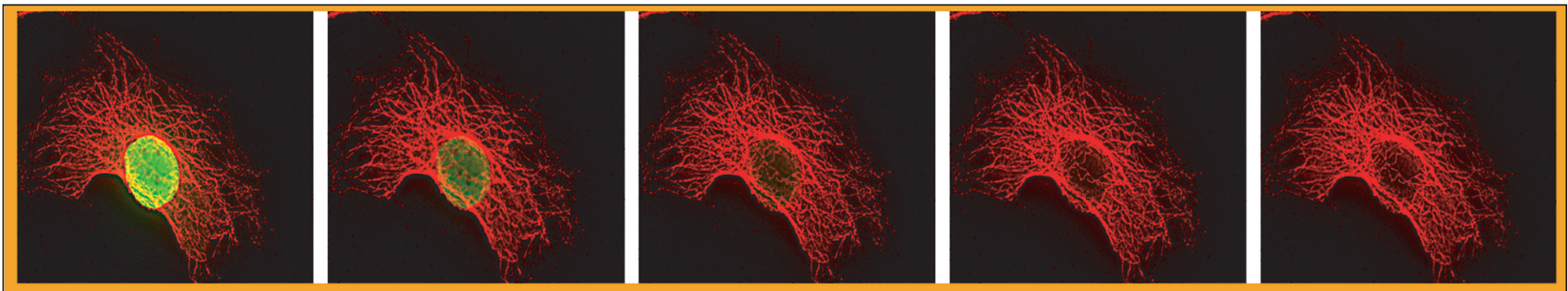

Figure 5. Nuclear antigens were stained with Alexa Fluor 488 (green), and microtubules were stained with Qdot streptavidin conjugate (red). The sample was continuously exposed for $3 \mathrm{~min}$ with light from a 100-W mercury lamp, $485 \pm 20 \mathrm{~nm}$ excitation filter, under a $60 \times / 1.40$ oil objective. Images were captured with a CCD camera and are shown for $0,20,60,120$, and $180 \mathrm{~s}$.

aware of any literature results that describe the use of quantum dot conjugates in multi-photon microscopy. This promises to be a valuable course for further investigation.

\section{EXAMPLES OF CELL AND TISSUE IMAGING WITH QUANTUM DOTS}

Quantum dot conjugates have been used to detect the breast cancer marker Her2 on fixed cells, mouse mammary tumor sections, and on the surface of unfixed live cells (20). The size of the quantum dots does not appear to have a signif- icant effect: intracellular targets, including microtubules, actin filaments, nuclei, and mitochondria have been effectively labeled (20) (Figures 4, 5, and 6).

In live cell imaging, high-intensity and/or improperly chosen excitation wavelengths may affect the cell viability. Quantum dots can be excited using much lower power sources, and the excitation wavelength can be chosen so as to reduce any known photo-induced cellular changes. Quantum dots have been injected inside living Xenopus embryos (9). The authors of this paper reported that they were able to inject single cells with quantum dots. By following the quantum dot fluorescence during embryo development, cell lineage could 


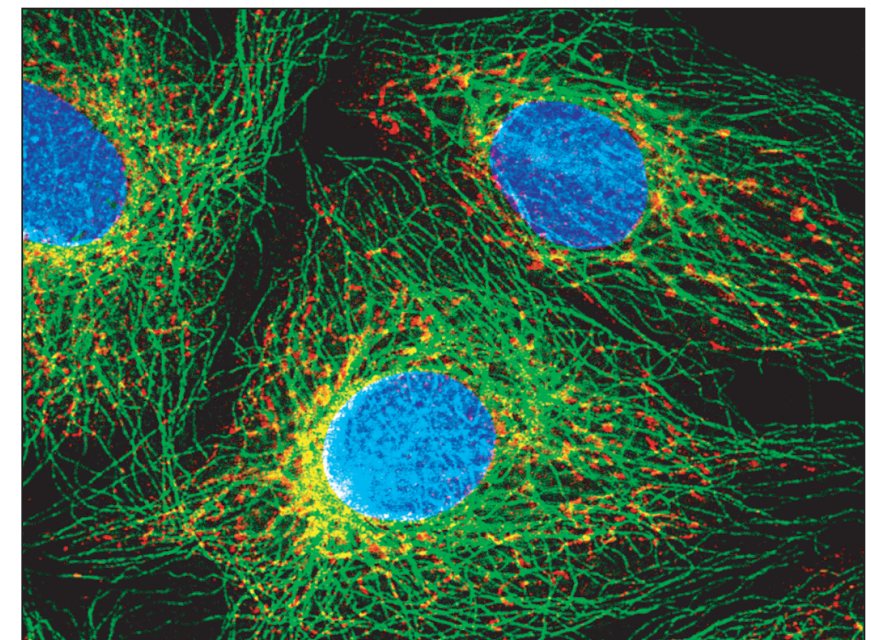

Figure 6. Microtubules (green) and mitochondria (red) in NIH 3 T3 mouse fibroblasts were stained with Qdot 525-streptavidin and Qdot 605-streptavidin. The nuclei were counterstained with Hoechst blue dye.

be followed all the way to the tadpole stage.

Endocytic uptake of quantum dots by cells has been demonstrated (12). In this study, the cells appeared unaffected by the presence of the quantum dots over a period of more than a week. Furthermore, the quantum dot fluorescence signal remained detectable throughout this period.

Quantum dots can be targeted to specific organs in vivo (1). Quantum dots conjugated to lung-targeting peptides were injected into mice and showed accumulation in the lungs. Quantum dots specific for blood vessels or lymphatic vessels in tumors were targeted in a similar manner. Several other applications have been reported $(16,19)$.

\section{THE FUTURE IS BRIGHT!}

Quantum dots conjugates exhibit extremely desirable properties for biological imaging. Their photostability makes them easy to use and allows integration of signal to increase sensitivity. Many spectrally narrow quantum dot colors can be created to allow simultaneous analysis of many conjugates. Quantum dots can be excited by the same light source, simplifying instrumentation. Quantum yield and excitation cross sections are very high, giving very bright signals. The large difference between excitation and emission colors reduces autofluorescence and simplifies instrumentation. Immediate improvements can be realized using quantum dots in current immunostaining applications. In the future, quantum dot conjugates promise to open up new applications in live cell and whole organism imaging. As further colors and conjugates of quantum dots become widely used, we anticipate a plethora of additional applications.

\section{REFERENCES}

1.Akerman, M.E., W.C. Chan, P. Laakkonen, S.N. Bhatia, and E. Ruoslahti. 2002. Nanocrystal targeting in-vivo. Proc. Natl. Acad. Sci. USA
99:12617-12621.

2.Alivisatos, A.P. 1996. Perspectives on the physical chemistry of semiconductor nanocrystals. J. Phys. Chem. B 100:13226-13239.

3.Alivisatos, A.P. 2001. Less is more in medicine. Sci. Am. 285:66-73.

4.Blanton, S.A., M.A. Hines, M.E. Schmidt, and P. Guyot-Sionnest. 1996. Two-photon spectroscopy and microscopy of II-VI semiconductor nanocrystals. J. Luminescence 70:253-268.

5.Bruchez, M., Jr., M. Moronne, P. Gin, S. Weiss, and A.P. Alivisatos. 1998. Semiconductor nanocrystals as fluorescent biological labels. Science 281:2013-2016.

6.Chan, W.C. and S. Nie. 1998. Quantum dot bioconjugates for ultrasensitive nonisotopic detection. Science 281:2016-2018.

7.Dabbousi, B.O., J. Rodriguez-Viejo, F.V. Mikulec, J.R. Heine, H. Mattoussi, R. Ober, K.F. Jensen, and M.G. Bawendi. 1997. (CdSe)ZnS core-shell quantum dots: synthesis and characterization of a size series of highly luminescent nanocrystallites. J. Phys. Chem. B 101:9463-9475.

8.Du, H., C. Chen, R. Krishnan, T.D. Krauss, J.M. Harbold, F.W. Wise,

M.G. Thomas, and J. Silcox. 2002. Optical properties of colloidal PbSe nanocrystals. Nano. Lett. 2:1321-1324.

9.Dubertret, B., P. Skourides, D.J. Norris, V. Noireaux, A.H. Brivanlou, and A. Libchaber. 2002. In vivo imaging of quantum dots encapsulated in phospholipid micelles. Science 298:1759-1762.

10.Empedocles, S. and M. Bawendi. 1999. Spectroscopy of single CdSe nanocrystallites. Acc. Chem. Res. 32:389-396.

11.Hines, M.A. and P. Guyot-Sionnest. 1996. Synthesis and characterization of strongly luminescing ZnS capped CdSe nanocrystals. J. Phys. Chem. 100:468-471.

12.Jaiswal, J.K., H. Mattoussi, J.M. Mauro, and S.M. Simon. 2002. Long-term multiple color imaging of live cells using quantum dot bioconjugates. Nat. Biotechnol. Online Dec 2, 2002 doi: 10.1038/nbt767.

13.Mattoussi, H., J.M. Mauro, E.R. Goldman, G.P. Anderson, V.C. Sundar, F.V. Mikulec, and M.G. Bawendi. 2000. Self-assembly of CdSe$\mathrm{ZnS}$ quantum dot bioconjugates using an engineered recombinant protein. J. Am. Chem. Soc. 122:12142-12150.

14.Murray, C.B., D.J. Norris, and M.G. Bawendi. 1993. Synthesis and characterization of nearly monodisperse CdE semiconductor nanocrystallites. J. Am. Chem. Soc. 115:8706-8715.

15.Norris, D.J. and M.G. Bawendi. 1996. Measurement and assignment of the size-dependent optical spectrum in CdSe quantum dots. Phys. Rev. B 53:16338-16346.

16.Parak, W.J., R. Boudreau, M. Le Gros, D. Gerion, D. Zanchet, C.M. Micheel, S.C. Williams, A.P. Alivisatos, and C. Larabell. 2002. Cell motility and metastatic potential studies based on quantum dot imaging of phagokinetic tracks. Adv. Mat. 14:882-885.

17.Peng, X., M.C. Schlamp, A.V. Kadavanich, and A.P. Alivisatos. 1997. Epitaxial growth of highly luminescent $\mathrm{CdSe} / \mathrm{CdS}$ core/shell nanocrystals with photostability and electronic accessibility. J. Am. Chem. Soc. 119:7019-7029.

18.Quantum Dot. 2002. Qdot 605 Streptavidin Conjugate User Manual. Quantum Dot, Hayward, CA.

19.Rosenthal, S.J., I. Tomlinson, E.M. Adkins, S. Schroeter, S. Adams, L. Swafford, J. McBride, Y. Wang, L.J. DeFelice, and R.D. Blakely. 2002. Targeting cell surface receptors with ligand-conjugated nanocrystals. J. Am. Chem. Soc. 124:4586-4594.

20.Wu, X, H. Liu, J. Liu, K.N. Haley, J.A. Treadway, J.P. Larson, N. Ge, F. Peale, and M.P. Bruchez. 2002. Immunofluorescent labeling of cancer marker Her2 and other cellular targets with semiconductor quantum dots. Nat. Biotechnol. 21:41-46.

Address correspondence to Andy Watson, Quantum Dot Corporation, 26118 Research Rd., Hayward, CA 94545, USA. e-mail:arwatson@qdots.com

Suggestions for contributions to the Biolmaging feature are welcomed by its editor, Dr. Steve Paddock (paddock@facstaff.wisc.edu) 